\title{
Dynamic Modeling and Analysis of Vertical Vibration Reduction System for Passenger Train
}

\author{
Shichang DONG*, Jianjun MENG*, Hao SONG*, Decang LI*, Caiyun SONG** \\ *Lanzhou JiaoTong University, Lanzhou 730070, China, E-mail: dscnjit@163.com \\ **Jinken College of Technology, Nanjing 211156, China, E-mail: 763352867@qq.com \\ crossref http://dx.doi.org/10.5755/j02.mech.25536
}

\section{Introduction}

A gap in the mechanical system will make the system produce collision and impact, which will affect the performance and safe operation of the mechanical system. For example, the impact between the wheels and the track in the running of high-speed trains intensifies the vibration of the trains and affects the running stability and comfort of the trains. Therefore, the study of vibro-impact is of great significance to reduce the collision, impact and abrasion of mechanical system, as the same time to improve the safety, lifetime and efficiency of the mechanical system. In recent years, the theory and application of vibro-impact system and gap system have made rapid progress [1-3].

The existing vehicle vertical vibration reduction system is mainly designed according to the track (road surface) irregularity $[4,5]$. Wei et al. [6] used the proposed dynamic model for a safety analysis and a vibrationreduction evaluation to theoretically validate the feasibility of semi-active magneto-rheological steel-spring FST. Cai et al. [7] studied the vibration control effect of long elastic sleeper track in subways though experiment. However, due to the existence of the wheel and rail gap, even if the train is running on completely smooth track, Chaos vibration will occur. There are many studies on wheel-rail impact and vehicle control [8-12]. Choi et al. [13] predicted the vibration of trains running on ballasted track by measuring the impact factor of track. However, these papers rarely analyze train vibration from the perspective of vibroimpact. In recent years, there are much theoretical research on vibro-impact [14-18]. Liu et al. [19] studied the dynamics of a capsule system in various friction environments. Yue et al. [20] focused on the coexistence of strange nonchaotic attractors (SNAs) and a novel mixed attractor in a periodically driven three-degree-of-freedom vibro-impact system with symmetry. However, few of them are combined with engineering practice. Therefore, in this paper, based on the wheel-rail impact vibration, the stiffness and natural damping of the carriage are considered. The dynamic model of the vertical vibration damping system of the train is built. The influence of different damping system parameters on the vibration of the running train is studied.

$$
\left[\begin{array}{ccc}
1 & 0 & 0 \\
0 & m_{2} & 0 \\
0 & 0 & m_{3}
\end{array}\right]\left\{\begin{array}{l}
\ddot{x}_{1} \\
\ddot{x}_{2} \\
\ddot{x}_{3}
\end{array}\right\}+\left[\begin{array}{ccc}
2 \zeta_{1} & -2 \zeta_{1} & 0 \\
-2 \zeta_{1} & 2 \zeta_{1}+2 \zeta_{2} & -2 \zeta_{2} \\
0 & -2 \zeta_{2} & 2 \zeta_{2}+2 \zeta_{3}
\end{array}\right]\left\{\begin{array}{l}
\dot{x}_{1} \\
\dot{x}_{2} \\
\dot{x}_{3}
\end{array}\right\}+\left[\begin{array}{ccc}
k_{1} & -k_{1} & 0 \\
-k_{1} & k_{1}+k_{2} & -k_{2} \\
0 & -k_{2} & k_{2}+k_{3}
\end{array}\right]\left\{\begin{array}{l}
x_{1} \\
x_{2} \\
x_{3}
\end{array}\right\}=\left\{\begin{array}{l}
f_{10} \\
f_{20} \\
f_{30}
\end{array}\right\} \sin (\omega t+\tau)
$$

$m_{4} \ddot{x}_{4}+2 \zeta_{4} \dot{x}_{4}+k_{4} x_{4}=f_{40} \sin (\omega t+\tau)$.

\section{System model}

Assuming that the passenger train's wheel-rail impact is a rigid impact and the motion in the vertical direction is considered only, the model of a single wheel vibro-impact system is shown in Fig. 1. The meanings of the symbols in Fig. 1 are shown in Table 1. Natural damping and carriage stiffness are connected to imaginary inertial spaces. When the displacement of the wheel minus the displacement of the rail is equal to the gap, wheel and rail impact. After impacting, wheel and rail get new speed and then impact again and again.

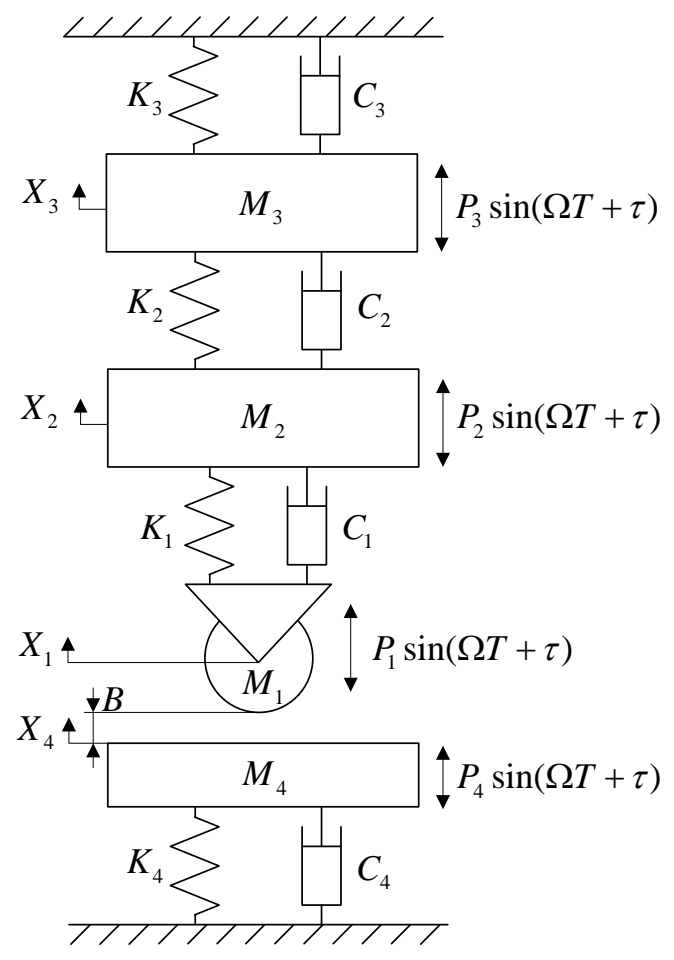

Fig. 1 Dynamic model of a single wheel vibro-impact system for passenger train

As shown in Fig. 1, the dimensionless differential equation where the system does not impact with each other can be written as:

In the formula, dimensionless quantities are: 
$m_{i}=\frac{M_{i}}{M_{1}}, k_{i}=\frac{K_{i}}{K_{1}}, f_{i 0}=\frac{P_{i}}{P_{0}}, \zeta_{i}=\frac{C_{i}}{2 \sqrt{K_{1} M_{1}}}, t=T \sqrt{\frac{K_{1}}{M_{1}}}, b=\frac{B K_{1}}{F_{0}}, \omega=\Omega \sqrt{\frac{M_{1}}{K_{1}}}, x_{i}=\frac{X_{i} K_{1}}{P_{0}}, i=1,2,3, P_{0}=P_{1}+P_{2}+P_{3}+P_{4}$

Table 1

The symbolic definition in Fig. 1

\begin{tabular}{|c|c|c|c|}
\hline Symbol & Definition & Symbol & Definition \\
\hline$M_{1}$ & Wheel mass & $C_{1}$ & Primary suspension damping \\
\hline$M_{2}$ & Bogie mass & $C_{2}$ & Secondary suspension damping \\
\hline$M_{3}$ & Partial body mass & $C_{3}$ & Natural damping \\
\hline$M_{4}$ & Track mass & $C_{4}$ & Track damping \\
\hline$K_{1}$ & Primary suspension stiffness & $P_{i} \sin (\Omega T+\tau)$ & Harmonic force \\
\hline$K_{2}$ & Secondary suspension stiffness & $X_{i}$ & Vertical displacement \\
\hline$K_{3}$ & Carriage stiffness & $B$ & Wheel/rail gap \\
\hline$K_{4}$ & Track stiffness & \multicolumn{2}{c}{} \\
\hline
\end{tabular}

When the gap is 0 , the motion equation of the wheel and rail is:

$$
\begin{aligned}
& \dot{x}_{1-}+m_{4} \dot{x}_{4-}=\dot{x}_{1+}+m_{4} \dot{x}_{4+}, \\
& R=\frac{\dot{x}_{4+}-\dot{x}_{1+}}{\dot{x}_{1-}-\dot{x}_{4-}} .
\end{aligned}
$$

The dimensionless instantaneous velocity before and after the impact of the wheel can be represented by $\dot{x}_{1-}$ and $\dot{x}_{1+}$ respectively. The dimensionless instantaneous velocity before and after the rail impact can be expressed by $\dot{x}_{4-}$ and $\dot{x}_{4+}$ respectively. $R$ is coefficient of restitution. Eqs (4) and (5) can be obtained:

$$
\begin{aligned}
& \dot{x}_{1+}=\frac{1-R m_{4}}{1+m_{4}} \dot{x}_{1-}+\frac{m_{4}(1+R)}{1+m_{4}} \dot{x}_{4-}, \\
& \dot{x}_{4+}=\frac{1+R}{1+m_{4}} \dot{x}_{1-}+\frac{m_{4}-R}{1+m_{4}} \dot{x}_{4-} .
\end{aligned}
$$

The semi-analytical solution of the system is then obtained through a series of derivation.

Select Poincare section: $\sigma$ where $\theta=\omega t$, and the Poincaré map of periodic motion of the system can be established by selecting the section:

$$
\Delta \mathbf{X}^{\prime}=f(\mathbf{v}, \Delta \mathbf{X})
$$

where: $X^{*}=\left(x_{10}, \dot{x}_{1+}, x_{20}, \dot{x}_{20}, x_{30}, \dot{x}_{3+}, x_{40}, \dot{x}_{4+}\right)^{T}, \mathbf{v} \in \mathrm{R}^{\mathbf{1}}$, are fixed points in the periodic collision motion process of the system on the Poincare section $\sigma, \Delta X$ and $\Delta X^{\prime}$ are the relevant disturbance quantity of the fixed point on the section $\sigma$.

When appropriate system parameters are selected, the symbol $q=p / n$ is usually used to represent the periodic motion of the vibro-impact system $(q=p / n$ does not represent the rational number in general, but only the symbol here), $n$ is used to represent the number of periods of force, and $p$ is used to represent the number of impacts. The periodic motion of $q=1 / n$ refers to: directly set the instantaneous time $t$ after the impact of the two oscillators $M_{1}$ and $M_{4}$ to be 0 . It is not difficult to find that the dimensionless time $t$ is just equal to $2 n \pi / \omega(n=1,2 \ldots)$ at the moment when the two oscillators $M_{1}$ and $M_{4}$ impact with each other. By shifting the origin of $\theta$ coordinate to a collision point, the boundary conditions of periodic motion of the system can be known:

$$
\begin{aligned}
& x_{10}=x_{1}(0)=x_{1}(2 n \pi / \omega), x_{1}(0)-x_{4}(0)=b \\
& \dot{x}_{1+}=\dot{x}_{1}(0)=\frac{1-R m_{4}}{1+m_{4}} \dot{x}_{1}(2 n \pi / \omega)+\frac{m_{4}(1+R)}{1+m_{4}} \dot{x}_{4}(2 n \pi / \omega) \\
& x_{20}=x_{2}(0)=x_{2}(2 n \pi / \omega), \dot{x}_{20}=\dot{x}_{2}(0)=\dot{x}_{2}(2 n \pi / \omega) \\
& \dot{x}_{4+}=\dot{x}_{4}(0)=\frac{1+R}{1+m_{4}} \dot{x}_{1}(2 n \pi / \omega)+\frac{m_{4}-R}{1+m_{4}} \dot{x}_{4}(2 n \pi / \omega) \\
& x_{30}=x_{3}(0)=x_{3}(2 n \pi / \omega), \dot{x}_{30}=\dot{x}_{3}(0)=\dot{x}_{3}(2 n \pi / \omega)
\end{aligned}
$$

Thus, the system parameters of the vibro-impact system moving in $q=1 / n$ period can be obtained, where $\dot{x}_{1}(2 n \pi / \omega)=\dot{x}_{1-}$ and $\dot{x}_{4}(2 n \pi / \omega)=\dot{x}_{4-}$ are the expressions of the impact velocity of the system. The linearized matrix (formal solution of Jacobian matrix) of Poincaré mapping at a fixed point can be written as:

$$
D f\left(v, X^{*}\right)=\left.\frac{\partial f(v, \Delta X)}{\partial \Delta X}\right|_{\left(v, X^{*}\right)} .
$$

\section{Dynamics simulation and analysis of the system}

According to the actual train parameters $\left(K_{1}\right.$ is between 0.2 and $1.5 \mathrm{MN} / \mathrm{m}, K_{2}$ is between 0.1 and $0.6 \mathrm{MN} / \mathrm{m}$, $C_{1}$ is between 10 and $30 \mathrm{kN} \cdot \mathrm{s} / \mathrm{m}, C_{2}$ is between 15 and 45 $\mathrm{kN} \cdot \mathrm{s} / \mathrm{m}$ ), the simulation parameters are selected as shown in the Table 2. The first column of the table is the variable symbols, the second column is the first set of parameter values, and the third column is the second set of parameter values. The second set of parameters differs from the first set in value $\zeta_{1}$ only.

Table 2

Simulation parameters

\begin{tabular}{|c|rll|rrr|}
\hline Symbols & \multicolumn{3}{|c|}{ Values 1} & \multicolumn{3}{|c|}{ Values 2} \\
\hline$m_{2}, m_{3}, m_{4}$ & 1.7 & 1.7 & 0.6 & 1.7 & 1.7 & 0.6 \\
\hline$k_{2}, k_{3}, k_{4}$ & 0.8 & 1.2 & 1.5 & 0.8 & 1.2 & 1.5 \\
\hline$\zeta_{2}, \zeta_{3}, \zeta_{4}$ & 1.2 & 1.5 & 0.5 & 1.2 & 1.5 & 0.5 \\
\hline$\zeta_{1} \cdot R \cdot b$ & 0.6 & 0.7 & 1.5 & 0.3 & 0.7 & 1.5 \\
\hline
\end{tabular}

Fig. 2 shows the Poincaré map generated after the 
simulation of the first set of parameters. Fig. 2a shows a single point on the Poincaré map. Fig. $2 \mathrm{~b}$ shows two points on the Poincaré map. Fig. $3 \mathrm{c}$ shows four points on the

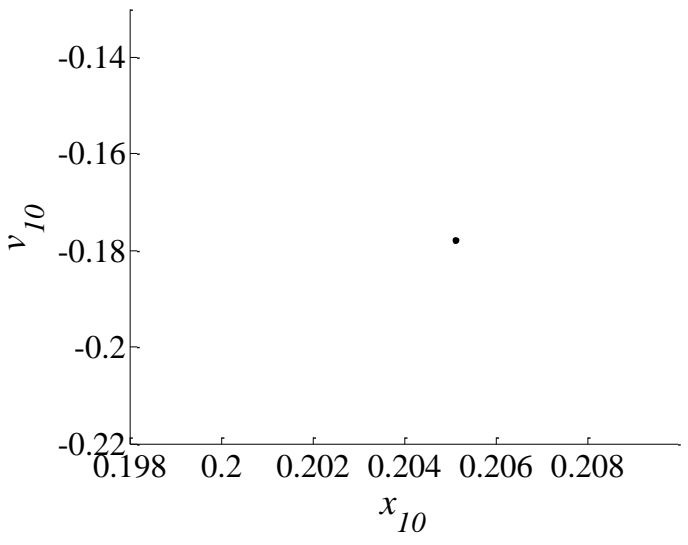

a) $\omega=2.23$

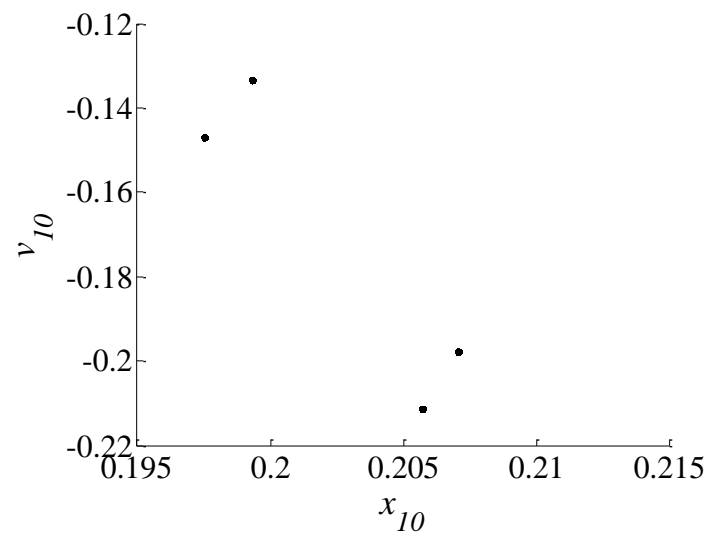

c) $\omega=2.249$
Poincaré map, and Fig. 2d shows an infinite number of points on the Poincaré map.

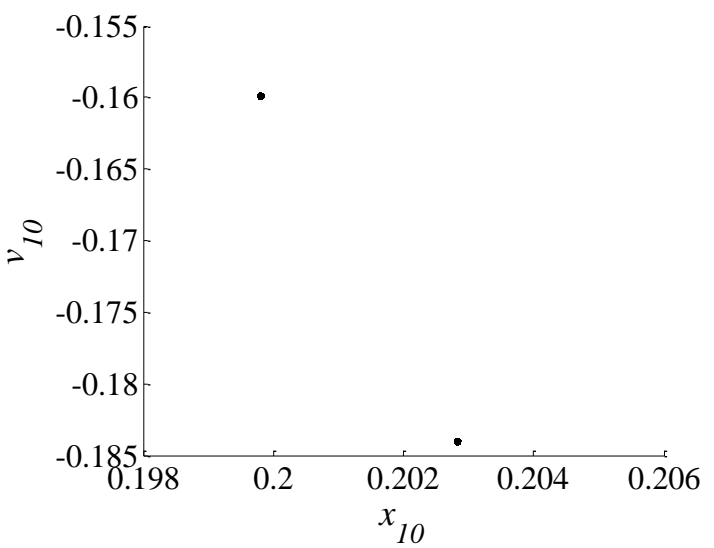

b) $\omega=2.2452$

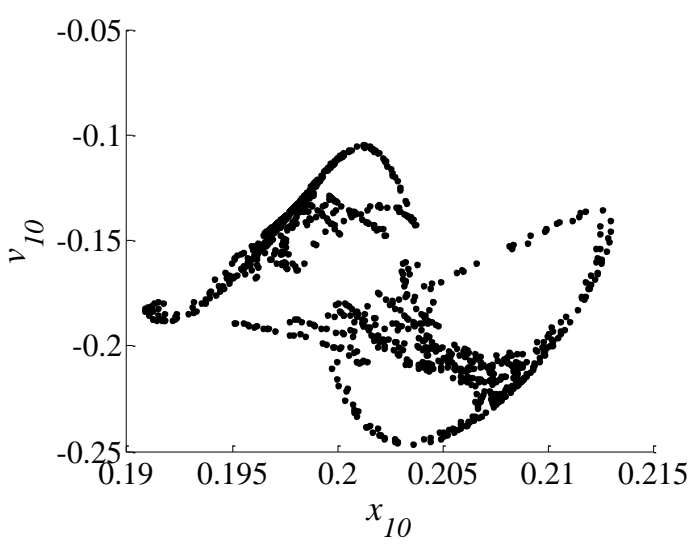

d) $\omega=2.252$

Fig. 2 Poincaré maps of the first set of parameters

Fig. 3 shows the acceleration time diagram formed after the simulation of the first parameter. As shown in Fig. 3, a, the acceleration presents a singleperiod characteristic, with its maximum value less than 0.4. As shown in Fig. 3, b, the acceleration presents a doubleperiodic characteristic with an amplitude of close to 0.4. As shown in Fig. 3, c, the acceleration presents a 4-period characteristic, whose amplitude is greater than 0.4. As shown in Fig. 3, d, the acceleration curve has no periodicity, and its amplitude is close to 0.5 .

Choosing the first set of parameters to the simulation, when $\omega<\omega_{c}=2.23$, the system has a stable periodic motion $q=1 / 1$, as shown in Fig. 2, a and Fig. 3, a. As $\omega$ increases, the system doubles the bifurcation as $q=2 / 2$ periodic motion, as shown in Fig. 2, b and Fig. 3, b. $\omega$ keeps going up to the $\omega=2.249$, the system doubles to periodic motion $q=4 / 4$, as shown in Fig. 2, c and Fig. 3, c. Finally, as $\omega$ continues to increase, the system goes into chaos motion, as shown in Fig. 2, d and Fig. 3, d.

The simulation results of the second set of parameters are shown in Fig. 4. Fig. 4, a and Fig. 4, c are Poincaré maps, Fig. 4, b and Fig. 4, d are acceleration time diagrams.

When the second set of parameters is selected for simulation, compared with the first set of parameters, only the value $\zeta_{1}$ of the system is reduced. The reduction of $\zeta_{1}$ also means that the damping of the primary suspension is reduced. By comparing Fig. 2, a with Fig. 4, a, it is found that the motion of the system changes from stable period 1 to period 4 when the value of $\zeta_{1}$ decreases with the constant $\omega$. By comparing Fig. 3, a and Fig. 4, b, it is also not difficult to find that the amplitude of wheel's acceleration increases with the decrease of $\zeta_{1}$.

By comparing Fig. 2, b with Fig. 4, c, it is found that the system doubles from 2 period to 4 period with the decrease of $\zeta_{1}$. Similarly, the amplitude of wheel's acceleration is also increased, as shown in Fig. 3, b and Fig 4, d.

\section{Engineering verification and discussion}

During the operation of the train, the mass of the whole train will change due to the change in the number of passengers. Track stiffness and damping will change due to the change of road conditions. As the temperature changes, the damping and stiffness of the carriage will change. The initial sensitivity of a chaotic system can cause a completely different vibration effect. Once, an abnormal vibration occurred in one carriage of the train, and the periodic amplitude of vertical acceleration of wheelset was found to increase during detection (The test point was placed on the wheelset bearings), as shown in Fig. 5. We thought there may have been structural damage to the wheels or the bearings and other bogie components, but no structural damage was found when the further 


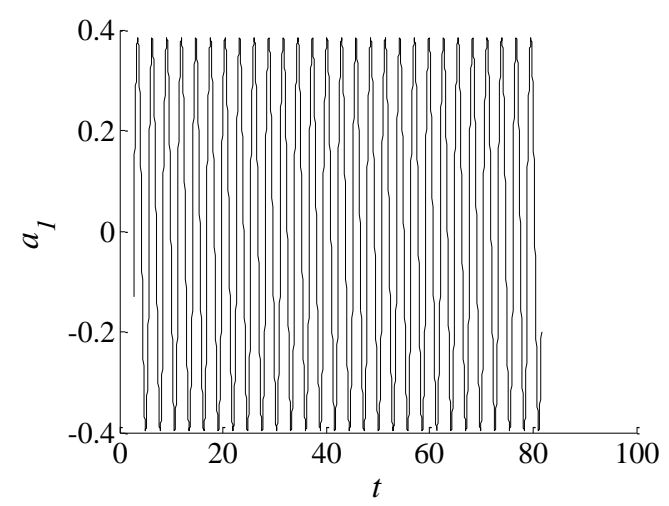

a) $\omega=2.23$

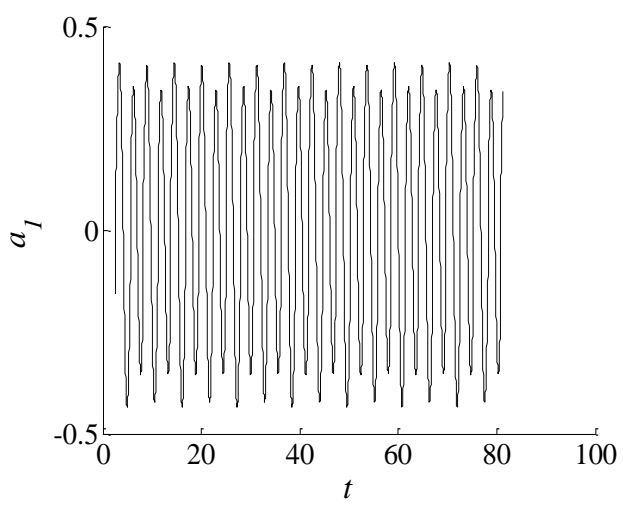

c) $\omega=2.249$

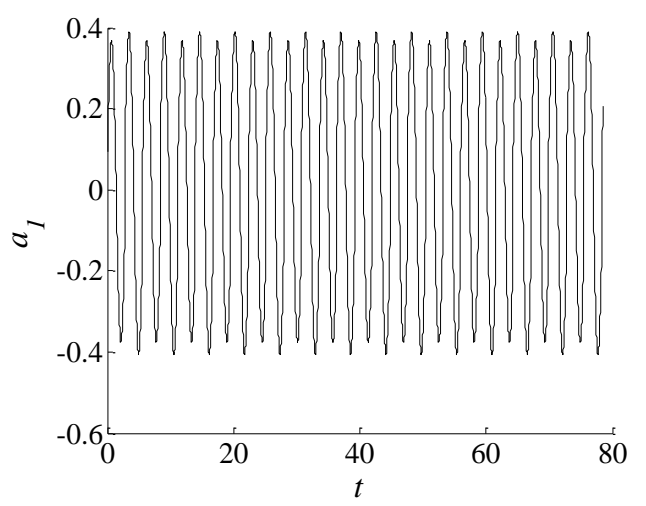

b) $\omega=2.2452$

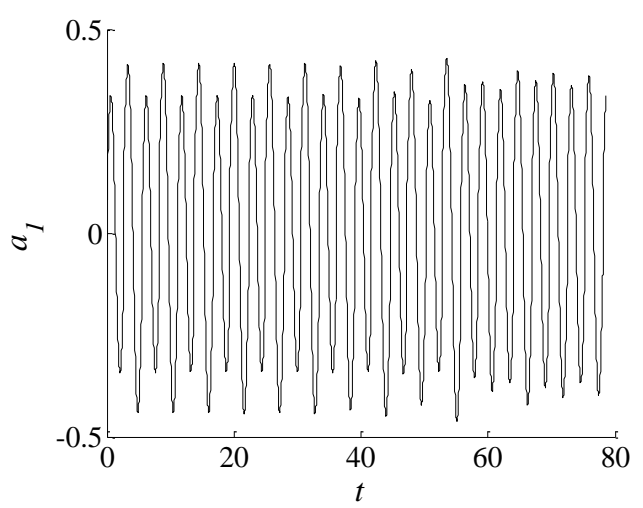

d) $\omega=2.252$

Fig. 3 Acceleration time diagrams of the first set of parameters

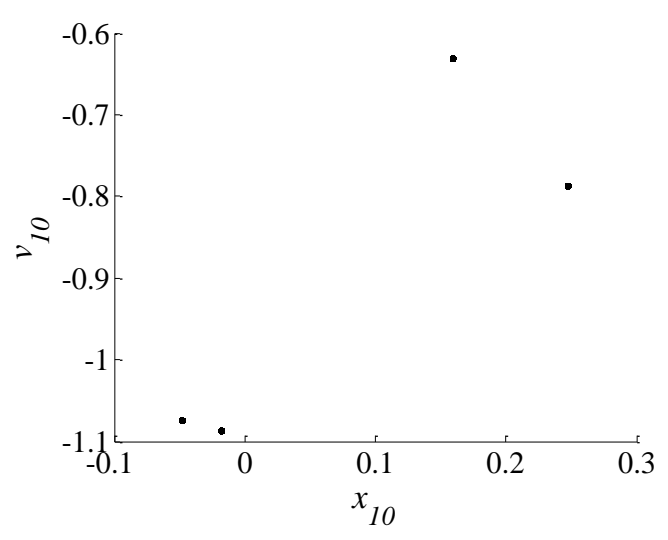

a) Poincaré map $\omega=2.23$

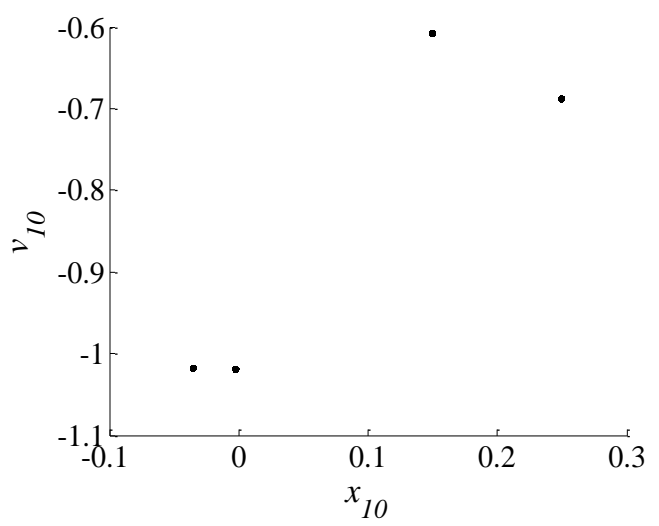

c) Poincaré map $\omega=2.245$

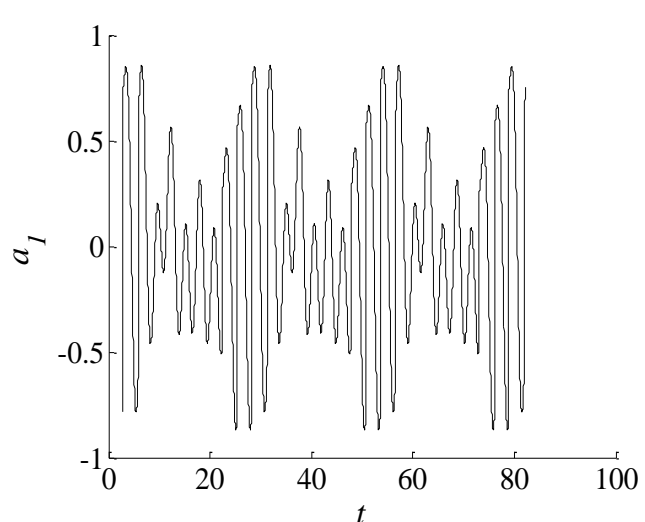

b) Acceleration time diagram $\omega=2.23$

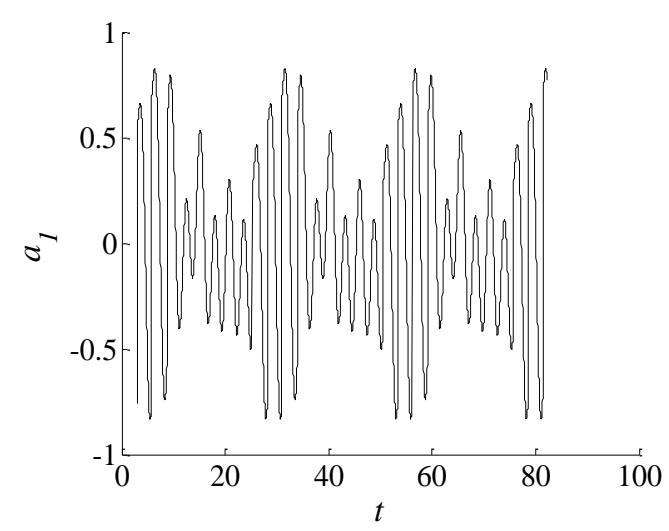

d) Acceleration time diagram $\omega=2.245$

Fig. 4 Simulation results of the second set of parameters 
inspection was conducted, so we suspected that the system had bifurcation. The primary suspension shock absorber of this car was exchanged with a new shock absorber. When the train was running again at the same speed of $60 \mathrm{~km} / \mathrm{h}$, no abnormal vibration occurred in this car. Fig. 6 shows the vertical acceleration of wheelset after the shock absorber was exchanged. Later we checked the old shock absorber and found that there was a slow leakage of oil which reduced the damping. This engineering practice verified the correctness of our simulation.

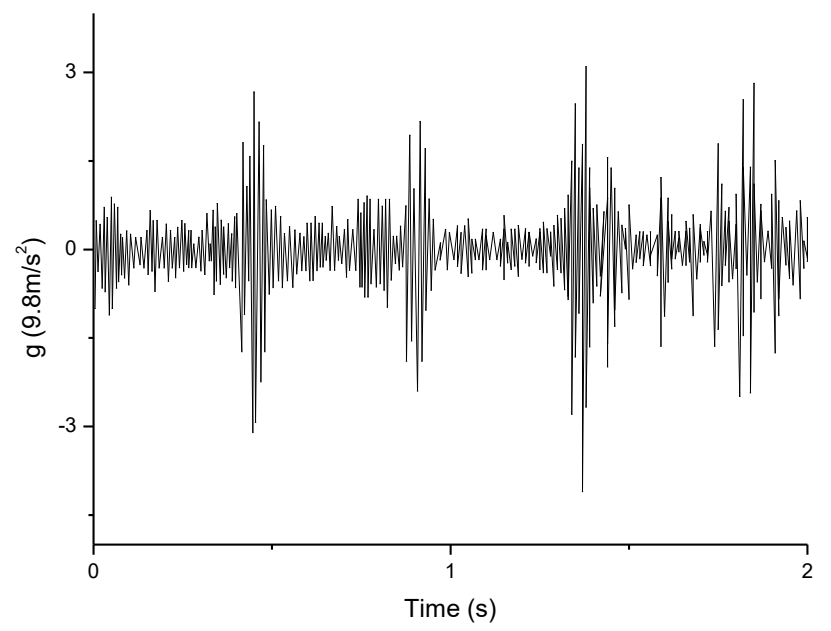

Fig. 5 Vertical vibration acceleration of wheelset of abnormal vibration carriage

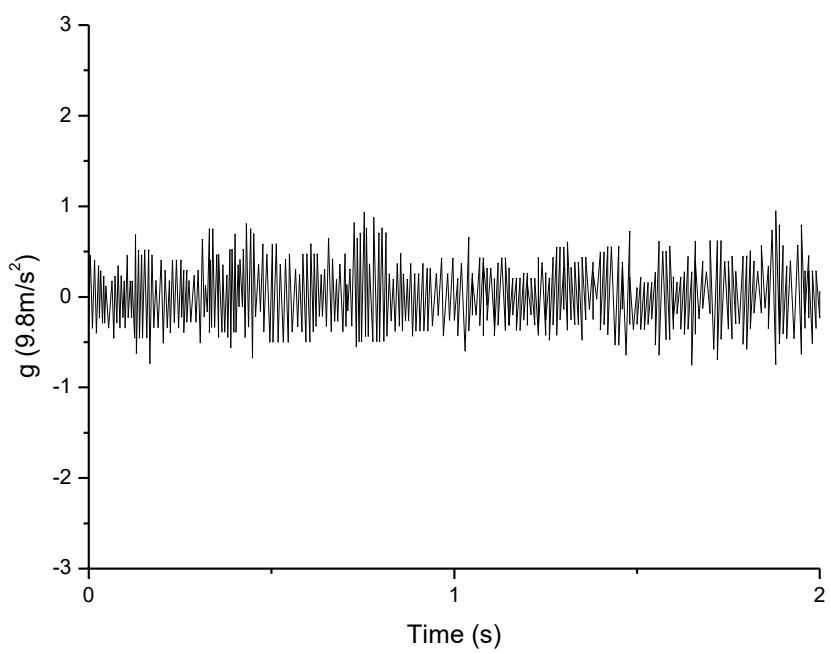

Fig. 6 Vertical vibration acceleration of wheelset after shock absorber replacement

\section{Conclusions}

This paper analyzes the vertical vibration damping system of the passenger train. The dynamic model of the passenger train's vertical vibration damping system is built as a four-degree-of-freedom vibro-impact system model. The simulation shows that the train may enter into a chaotic state in the actual operation process. The engineering practice shows that the vertical vibration of the train will lose its stability with the decrease of primary suspension damping, and the simulation results are also verified. It has been proved that the vibration reduction design of the train should consider not only the track unevenness, but also the multi-period vibration, even chaos vibration caused by wheel-rail vibro-impact.

\section{Acknowledgements}

This study was funded by the National Natural Science Foundation of China (no. 62063013 and no. 72061021) and Science Research Funding Project of Nanjing Institute of Technology (no. QKJ201713).

\section{References}

1. Luo, G.; Ma, L.; Lv, X. 2009. Dynamic analysis and suppressing chaotic impacts of a two-degree-offreedom oscillator with a clearance, Nonlinear Analysis: Real World Applications 10(2): 756-778. http://doi.org/https://doi.org/10.1016/j.nonrwa.2007.11. 002.

2. Niu, J.; Liu, R.; Shen, Y.; Yang, S. 2019. Stability and bifurcation analysis of single-degree-of-freedom linear vibro-impact system with fractional-order derivative, Chaos, Solitons \& Fractals 123: 14-23. http://doi.org/10.1016/j.chaos.2019.03.035.

3. Zhang, Y.; Luo, G. 2018. Multistability of a threedegree-of-freedom vibro-impact system, Communications in Nonlinear Science and Numerical Simulation 57: 331-341. http://doi.org/10.1016/j.cnsns.2017.10.007.

4. Saglam, F.; Unlusoy, Y. S. 2016. Adaptive ride comfort and attitude control of vehicles equipped with active hydro-pneumatic suspension, International Journal of Vehicle Design 71(1/2/3/4): 31-51. http://doi.org/10.1504/IJVD.2016.078764.

5. Wang, Q.; Zeng, J.; Wei, L.; Zhou, C.; Zhu, B. 2017. Reduction of vertical abnormal vibration in carbodies of low-floor railway trains by using a dynamic vibration absorber, Proceedings of the Institution of Mechanical Engineers Part F Journal of Rail and Rapid Transit 232(5): 1437-1447. http://doi.org/10.1177/0954409717731234.

6. Wei, K.; Zhao, Z.; Du, X.; Li, H.; Wang, P. 2019. A theoretical study on the train-induced vibrations of a semi-active magneto-rheological steel-spring floating slab track, Construction and Building Materials 204: 703-715. http://doi.org/10.1016/j.conbuildmat.2019.01.210.

7. Cai, X.; Li, D.; Zhang, Y.; Miao, Q.; Cui, R. 2018. Experimental Study on the Vibration Control Effect of Long Elastic Sleeper Track in Subways, Shock and Vibration 2018(526): 1-13. http://doi.org/10.1155/2018/6209518.

8. Bian, J.; Gu, Y.; Murray, M. H. 2013. A dynamic wheel-rail impact analysis of railway track under wheel flat by finite element analysis, Vehicle System Dynamics 51(6): 784-797. http://doi.org/10.1080/00423114.2013.774031.

9. Li, Q.; Thompson, D. J.; Toward, M. G. 2017. Estimation of track parameters and wheel-rail combined roughness from rail vibration, Proceedings of the Institution of Mechanical Engineers Part F Journal of Rail and Rapid Transit 232(4): 1149-1167. http://doi.org/10.1177/0954409717710126.

10. Naeimi, M.; Zakeri, J. A.; Shadfar, M.; Esmaeili, M. 2015. 3D dynamic model of the railway wagon to obtain the wheel-rail forces under track irregularities, Proceedings of the Institution of Mechanical Engineers Part K Journal of Multi-body Dynamics 229(4): 154- 
157.

http://doi.org/10.1177/1464419314566833.

11. Torstensson, P. T.; Squicciarini, G.; Krüger, M.; Pålsson, B. A.; Thompson, D. J. 2019. Wheel-rail impact loads and noise generated at railway crossings Influence of vehicle speed and crossing dip angle, Journal of Sound and Vibration 456: 119-136. http://doi.org/10.1016/j.jsv.2019.04.034.

12. Meng, J.; Xu, R.; Li, D.; Chen, X. 2019. Combining the matter-element model with the associated function of performance indices for automatic train operation algorithm, IEEE Transactions on Intelligent Transportation Systems 20: 253-263. http://doi.org/10.1109/TITS.2018.2805917.

13. Choi, J. 2013. Prediction of displacement induced by tilting trains running on ballasted tracks through measurement of track impact factors, Engineering Failure Analysis 31: 360-374. http://doi.org/10.1016/j.engfailanal.2013.02.004.

14. Haiyan, H. 1995. Controlling chaos of a periodically forced nonsmooth mechanical system, Acta Mechanica Sinica 11(3): 251-258. http://doi.org/10.1007/BF02487728.

15. Leine, R. I. 2012. Non-smooth stability analysis of the parametrically excited impact oscillator, International Journal of Non-Linear Mechanics 47(9): 1020-1032. http://doi.org/10.1016/j.ijnonlinmec.2012.06.010.

16. Luo, G.; Lv, X. 2008. Dynamics of a plastic-impact system with oscillatory and progressive motions, International Journal of Non-Linear Mechanics 43(2): 100110. http://doi.org/10.1016/j.ijnonlinmec.2007.10.008.

17. Wen; Guilin. 2005. Criterion to identify Hopf bifurcations in maps of arbitrary dimension, Physical Review E Statal Nonlinear \& Soft Matter Physics 72(2): 026201 . http://doi.org/10.1103/PhysRevE.72.026201.

18. Xie, J.; Ding, W. 2005. Hopf-Hopf bifurcation and invariant torus T2 of a vibro-impact system, International Journal of Non-Linear Mechanics 40(4): 531-543. http://doi.org/10.1016/j.ijnonlinmec.2004.07.015.

19. Liu, Y.; Pavlovskaia, E.; Hendry, D.; Wiercigroch, M. 2013. Vibro-impact responses of capsule system with various friction models, International Journal of Mechanical Sciences 72(72): 39-54.

http://doi.org/10.1016/j.ijmecsci.2013.03.009.

20. Yue, Y.; Miao, P.; Xie, J. 2017. Coexistence of strange nonchaotic attractors and a special mixed attractor caused by a new intermittency in a periodically driven vibro-impact system, Nonlinear Dynamics 87(2): 11871207. http://doi.org/10.1007/s11071-016-3109-2.

\section{S. Dong, J. Meng, H. Song, D. Li, C. Song}

\section{DYNAMIC MODELING AND ANALYSIS OF VERTICAL VIBRATION REDUCTION SYSTEM FOR PASSENGER TRAIN}

S u m m a r y

Based on wheel-rail impact vibration and considering the carriage stiffness and natural damping, this paper builds a four-degree-of-freedom vibro-impact system model for passenger train's vertical vibration reduction system. The Poincaré map of the system is determined by the analytic solution of the system derived from the motion differential equation of the multi-degree-of-freedom vibroimpact system combined with Newton's second law. It is found that with the increase of excitation frequency, the system enters into chaotic motion by doubling bifurcation. It is also found that when the excitation frequency of the system is constant, the reduction of the primary suspension damping will also lead the system into an unstable state. On this basis, the dynamic parameters of the train are optimized to avoid Chaos in the train operation, reduce the vertical vibration of the train, improve the stability and comfort of the train operation.

Keywords: vibro-impact, train, vibration reduction, Poincaré map, Chaos.

Received September 11, 2020

Accepted October 04, 2021 